\title{
Warum Diabetes zwischen arm und reich unterscheidet
}

In wohlhabenden Nationen erkranken Personen mit geringem sozioökonomischem Status überdurchschnittlich häufig an Diabetes. Fast 50\% dieser sozialen Differenz können einer prospektiven Kohortenstudie zufolge durch Unterschiede in Lebensstil und Body-Mass-Index (BMI) erklärt werden.

Dass Risikofaktoren für einen Diabetes wie Bewegungsmangel, Adipositas, ungesunde Ernährung und Rauchen in sozial schwachen Gruppen stärker verbreitet sind, ist nichts Neues. Aber in älteren Studien ließ sich nur etwa ein Drittel der erhöhten

Diabetesinzidenz auf den ungesünderen Lebensstil zurückführen - möglicherweise weil er oft nur zu Studienbeginn untersucht wurde. Der Beitrag von modifizierbaren Risikofaktoren ist nämlich deutlich größer, wenn sie - wie in der Whitehall-IIStudie geschehen - über mehrere Jahre hinweg erfasst werden. Alle 7237 anfangs stoffwechselgesunde Studienteilnehmer im Alter zwischen 35 und 55 Jahre waren britische Staatsangestellte, deren Status je nach Position als hoch (Verwaltungsbeamte), mittel (z. B. leitende Angestellte) oder niedrig (z. B. Schreibkräfte) definiert wurde.

Sozial Schwache häufiger übergewichtig, aber...

Während der mittleren Beobachtungszeit von 14 Jahren wurde bei 818 Personen ein Diabetes diagnostiziert. Die Beamten mit den schlechtesten Positionen hatten ein um 86\% höheres Erkrankungsrisiko als Beamte mit Spitzenpositionen. Wenn Lebensstil und BMI bei Studienbeginn verglichen wurden, ließen sich damit 33\% der Differenz erklären. Ihr Anteil erhöhte sich auf 45\%, wenn mehrere Messungen und die Langzeitexposition gegenüber diesen Faktoren berücksichtigt wurden. Höhere Blut-

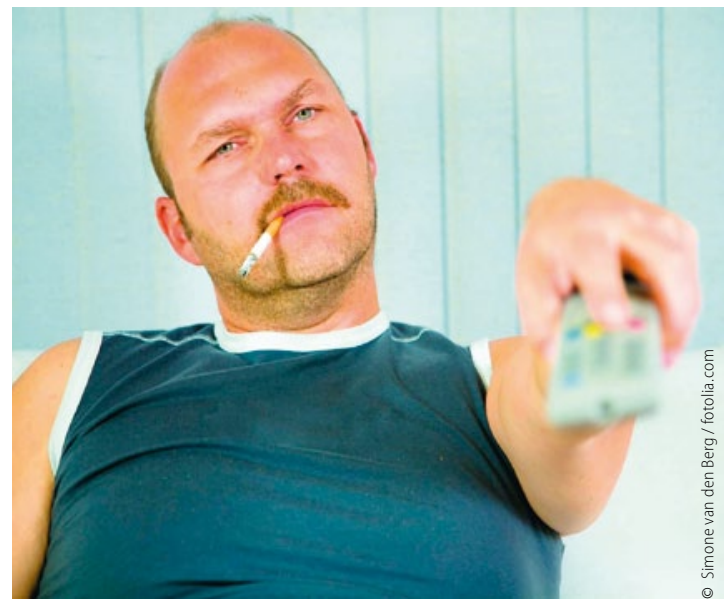

Menschen mit prekärer sozialer Position erkranken öfter an Diabetes.

fett- und Blutdruckwerte bei Studienbeginn waren für weitere $8 \%$ des Zusatzrisikos der beruflich niedriger gestellten Personen verantwortlich. Am stärksten trug jedoch mit $20 \%$ ein hoher BMI zu ihrer Gefährdung bei.

\section{...Was ist mit der anderen Hälfte des sozialen Gefälles?}

Immerhin noch knapp 50\% des sozialen Gefälles bei der Diabetesinzidenz lassen sich durch keinen der untersuchten Parameter erklären. Als weitere Mittler des hohen Diabetesrisikos von sozial schwächeren Personen kommen z. B. psychosoziale oder psychische Beeinträchtigungen, ungenügende Aufklärung oder eine schlechtere medizinische Versorgung infrage. Dafür gibt es jedoch bisher keine Belege.

Die Studie macht deutlich, dass das erhöhte Diabetesrisiko von gesellschaftlich unterprivilegierten Gruppen durch einen gesünderen Lebensstil inklusive eines normalen BMI zumindest deutlich reduziert werden könnte. Hier seien weitere Anstrengungen nötig, so die Autoren, um diese Risikofaktoren endlich besser in den Griff zu bekommen.

Dr. Beate Schumacher

Quelle: springermedizin.de basierend auf: Stringhini S et al. Contribution of modifiable risk factors to social inequalities in type 2 diabetes. BMJ 2012; online 21. August; doi: 10.1136/bmj.e5452

\section{Weltweite Analyse: Jeder Zehnte stirbt zu früh, weil er sich zu wenig bewegt}

Ob Diabetes, KHK, Darm- oder Brustkrebs - weltweit ließen sich bis zu 10\% solcher Erkrankungen durch mehr körperliche Aktivität verhindern. Die Lebenserwartung würde sich so um über acht Monate erhöhen. Sport ist Mord - an diesem Vorurteil dürfte kaum noch jemand glauben, zu sehr hat sich körperliche Aktivität als Bestandteil von Präventionsmaßnahmen etabliert. Präventionsmediziner um Dr. I-Min Lee von der Harvard Medical School in Boston berechneten nun, welcher Anteil bei den wichtigsten nicht übertragbaren Erkrankungen auf Bewegungsmangel zurückzuführen ist und wie es sich auswirken würde, wenn alle Menschen mindestens die von der WHO empfohlenen 150 Minuten pro Woche an leichter bis moderater (Wandern, gemütliches Radeln, Spazierengehen) oder 75 Minuten intensiver Aktivität wie Joggen absolvieren würden. Die Forscher griffen sowohl auf Erhebungen der WHO für die Länder zurück, ebenso auf Metaanalysen von epidemiologischen Studien. Berücksichtig wurden dabei etwa der Anteil körperlich Inaktiver in der Bevölkerung, deren Anteil bei den verschiedenen Erkrankungen und bei den Gesunden sowie das Risiko, durch Bewegungsmangel zu erkranken. Einige der Ergebnisse: Weltweit ist etwa ein Drittel $(35,2 \%)$ körperlich inaktiv, erfüllt also nicht die WHO-Kriterien. Bei innen sind die Raten für KHK um 33\%, für Typ-2-Diabetes um 63\%, für Brustkrebs um 34\% sowie für Darmkrebs um 43\% erhöht. Die Gefahr, verfrüht zu sterben, ist ebenfalls um $43 \%$ höher als bei aktiven Personen. Daraus berechneten die Forscher, dass knapp 6\% aller KHK-, 7\% aller Diabetes- sowie jeweils 10\% der Brustund Darmkrebserkrankungen auf mangelnde Bewegung zurückzuführen sind. Inaktivität ist der Grund für jeden zehnten Todesfall, der vor Erreichen der statistischen Lebenserwartung auftritt. Weltweit würde sich die Lebenserwartung um 0,7 Jahre erhöhen, wenn niemand mehr inaktiv wäre, so das Fazit der Forscher.

Thomas Müller

Quelle: springermedizin.de basierend auf: Lee IM et al. Effect of physical inactivity on major non-communicable diseases worldwide: an analysis of burden of disease and life expectancy. Lancet 2012, online 18. Juli 